\title{
A new path to first light for the Magdalena Ridge Observatory Interferometer
}

\author{
M. J. Creech-Eakman ${ }^{a}$, V. Romero ${ }^{a}$, I. Payne ${ }^{a}$, C. A. Haniff ${ }^{b}$, D. F. Buscher ${ }^{b}$, J. S. Young ${ }^{b}$, R. Cervantes ${ }^{a}$, C. \\ Dahl $^{a}$, A. Farris ${ }^{a}$, M. Fisher ${ }^{b}$, P. Johnston ${ }^{a}$, D. Klinglesmith ${ }^{a}$, H. Love ${ }^{a}$, D. Ochoa ${ }^{a}$, A. Olivares ${ }^{a}$, J. Pino ${ }^{a}$, C. \\ Salcido ${ }^{a}$, F. Santoro ${ }^{a}$, L. Schmidt ${ }^{c}$, E. B. Seneta ${ }^{b}$, X. Sun ${ }^{b}$, L. Jenka ${ }^{a}$, R. Kelly ${ }^{a}$, J. Price ${ }^{a}$, A. Rea ${ }^{b}$, J. Riker ${ }^{a}$, S. \\ Rochelle $^{a}$ \\ a - New Mexico Institute of Mining and Technology, Magdalena Ridge Observatory Interferometer, Socorro, NM, \\ USA, 87801 \\ b - Cavendish Laboratory, Cambridge University, Cambridge, UK, CB3 OHE \\ c - Department of Physics and Astronomy, Texas A\&M, College Station, TX 77843
}

Keywords: optical and infrared interferometry; interferometric imaging; fringe tracking; delay lines; telescopes; enclosures; space situational awareness

\begin{abstract}
:
The Magdalena Ridge Observatory Interferometer (MROI) was the most ambitious infrared interferometric facility conceived of in 2003 when funding began. Today, despite having suffered some financial short-falls, it is still one of the most ambitious interferometric imaging facilities ever designed. With an innovative approach to attaining the original goal of fringe tracking to $\mathrm{H}=14^{\text {th }}$ magnitude via completely redesigned mobile telescopes, and a unique approach to the beam train and delay lines, the MROI will be able to image faint and complex objects with milliarcsecond resolutions for a fraction of the cost of giant telescopes or space-based facilities. The design goals of MROI have been optimized for studying stellar astrophysical processes such as mass loss and mass transfer, the formation and evolution of YSOs and their disks, and the environs of nearby AGN.

The global needs for Space Situational Awareness (SSA) have moved to the forefront in many communities as Space becomes a more integral part of a national security portfolio. These needs drive imaging capabilities ultimately to a few tens of centimeter resolution at geosynchronous orbits. Any array capable of producing images on faint and complex geosynchronous objects in just a few hours will be outstanding not only as an astrophysical tool, but also for these types of SSA missions. With the recent infusion of new funding from the Air Force Research Lab (AFRL) in Albuquerque, NM, MROI will be able to attain first light, first fringes, and demonstrate bootstrapping with three telescopes by 2020 .

MROI's current status along with a sketch of our activities over the coming 5 years will be presented, as well as clear opportunities to collaborate on various aspects of the facility as it comes online. Further funding is actively being sought to accelerate the capability of the array for interferometric imaging on a short time-scale so as to achieve the original goals of this ambitious facility




\section{Introduction:}

The Magdalena Ridge Observatory Interferometer (MROI) is the most-ambitious optical interferometer currently under construction today. When completed, it will consist of 101.4 meter diameter altitudealtitude telescopes laid out in an equilateral-Y array of 28 pads spanning telescope separations from 7.8 to 346 meters (see figure 1). Fringe-tracking will occur at infrared wavelengths, while science will be accomplished at either infrared or optical wavelengths. The MROI will be capable of a vigorous science program as well as support of national security initiatives, as discussed in Young et al. $(2012)^{1}$ and briefly below.

The MROI active construction today is due to a recent successful proposal for funding from the AFRL. This funding is specifically for mitigating ground-based technical issues associated with imaging GEO assets with optical interferometers. This funding is being provided via a cooperative agreement over a five-year period and will allow MROI to deploy 3 telescopes on the array to phase track at sensitivities of $\mathrm{H}_{\text {mag }}=9.5$ and demonstrate bootstrapping needed for imaging of geosynchronous objects. Funding is anticipated to be provided in a flat profile of $\$ 5 \mathrm{M} /$ year for each year of the five-year cooperative agreement period. The funding is additionally permitting us to expand our collaboration at MROI to include NRAO, Lockheed-Martin, Applied Technology Associates (ATA) and several subcontractors in order to address specific issues of interest to the GEO imaging community interested in Space Situational Awareness (SSA) issues.

Included in the plans for these immediate five years are undertaking studies to provide answers to questions associated with: 1 ) the best approaches for algorithms used in imaging GEO assets either employing current or improved data reduction and aperture synthesis techniques, 2) examining the potential to fuse optical/infrared and radio data on a GEO asset to improve knowledge of these objects, and 3) evaluating current interferometric technology to determine what is feasible for use on a space interferometric facility and where improvements need to be made to propel the field forward.

Below is a brief update on the status and immediate plans for each of the major subsystems presently under development at MROI.

\section{Recent Progress on Interferometer Subsystems}

Below we present a brief discussion of each of the major subsystems of the facility that we have been making progress on since the last SPIE meeting in 2014. For general descriptions of each system, a reference paper will be given. Recent overall descriptions of MROI can be found in Santoro et al. (2012) and Buscher et al. (2013) . 


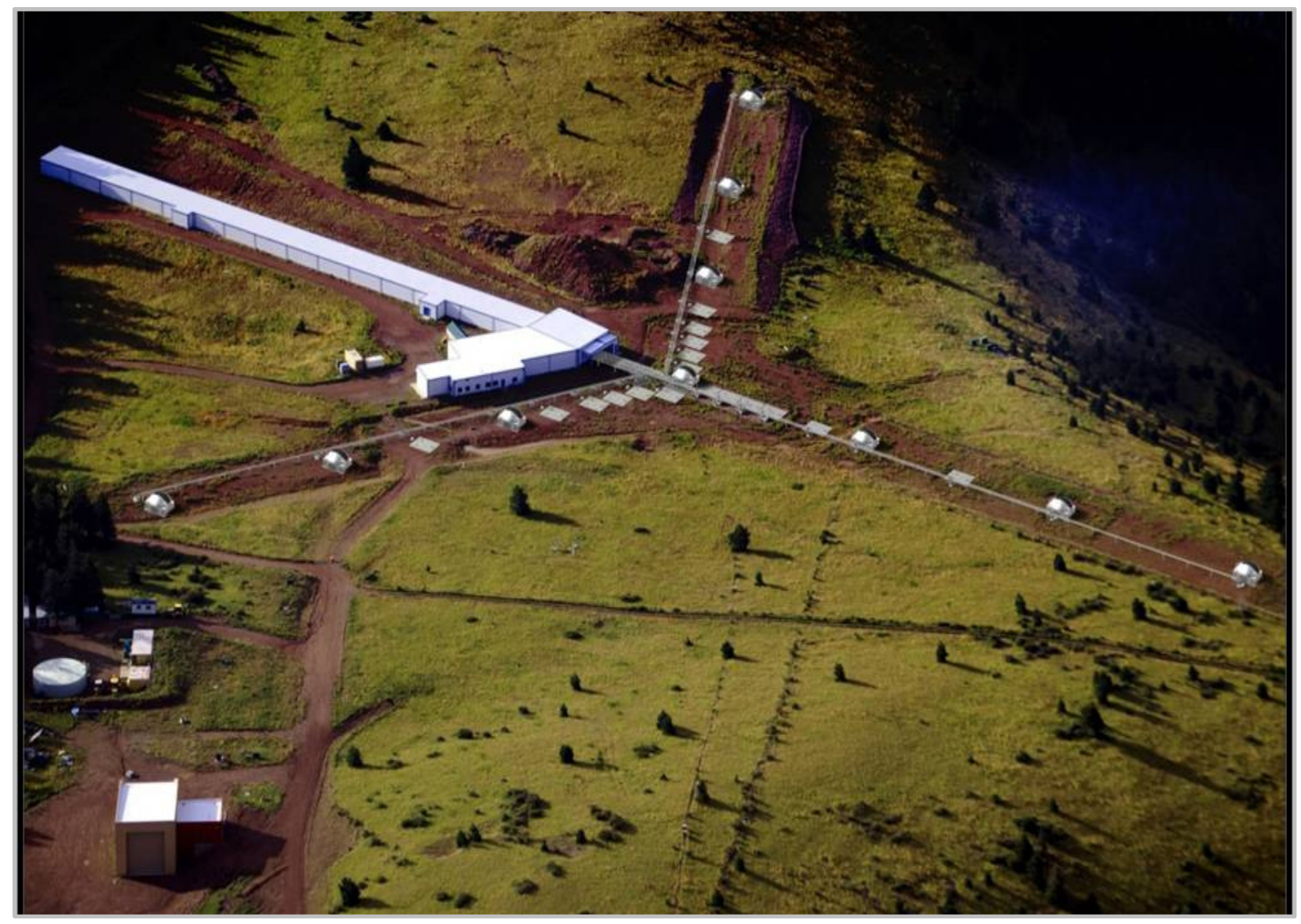

Figure 1: Photo of the completed MROI beam combining facility taken Fall 2015. In the lower left of the foreground is the Visitor's Center and Maintenance Facility where telescope mount \#1 is currently undergoing Site Acceptance Testing. Overlayed on the photo of the array are superimposed pads, telescopes and a beam relay system rendered by lead opto-mechanical MROI engineer A. Olivares and based on the actual design of the MROI infrastructure. The photo of the site courtesy of T. Eakman.

\subsection{Unit Telescope Mount}

Designed and built by Advanced Mechanical and Optical Systems (AMOS), the Unit Telescope Mount (UTM) is a polarization preserving, altitude-altitude design which delivers a $63 \mathrm{~nm}$ rms wavefront into the beam relay vacuum system ${ }^{4}$. In 2015 the Visitor's Center and Maintenance Facility (VCMF) at Magdalena Ridge was completed and released to the observatory from the NM Department of Transportation who funded the project. Included in the maintenance portion of the VCMF is a telescope interface platform and associated infrastructure needed to power and operate a telescope and its subsystems. The kinematic platform mount is located near a very tall garage door, allowing for a $25 \times 40$ degree view of the sky for acquiring starlight with the telescope. The first UTM, received at the NMT campus in late 2014, is presently being installed in the VCMF after which time it will undergo Site 
Acceptance Testing (SAT). We anticipate SAT being completed by late August, 2016. See figures 2 and 3 below.

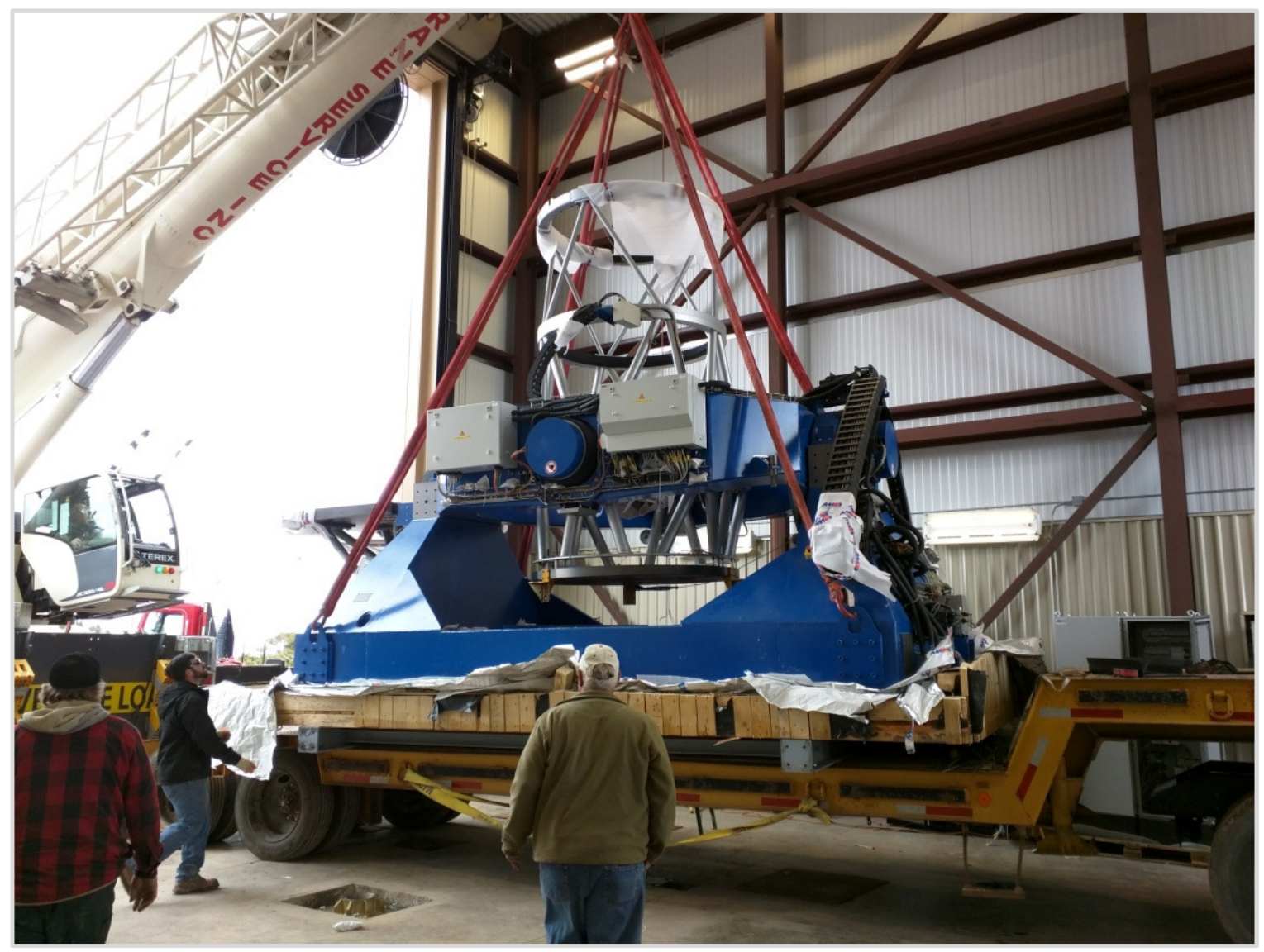

Figure 2: UTM being unloaded in the VCMF prior to installation on the kinematic mount. 


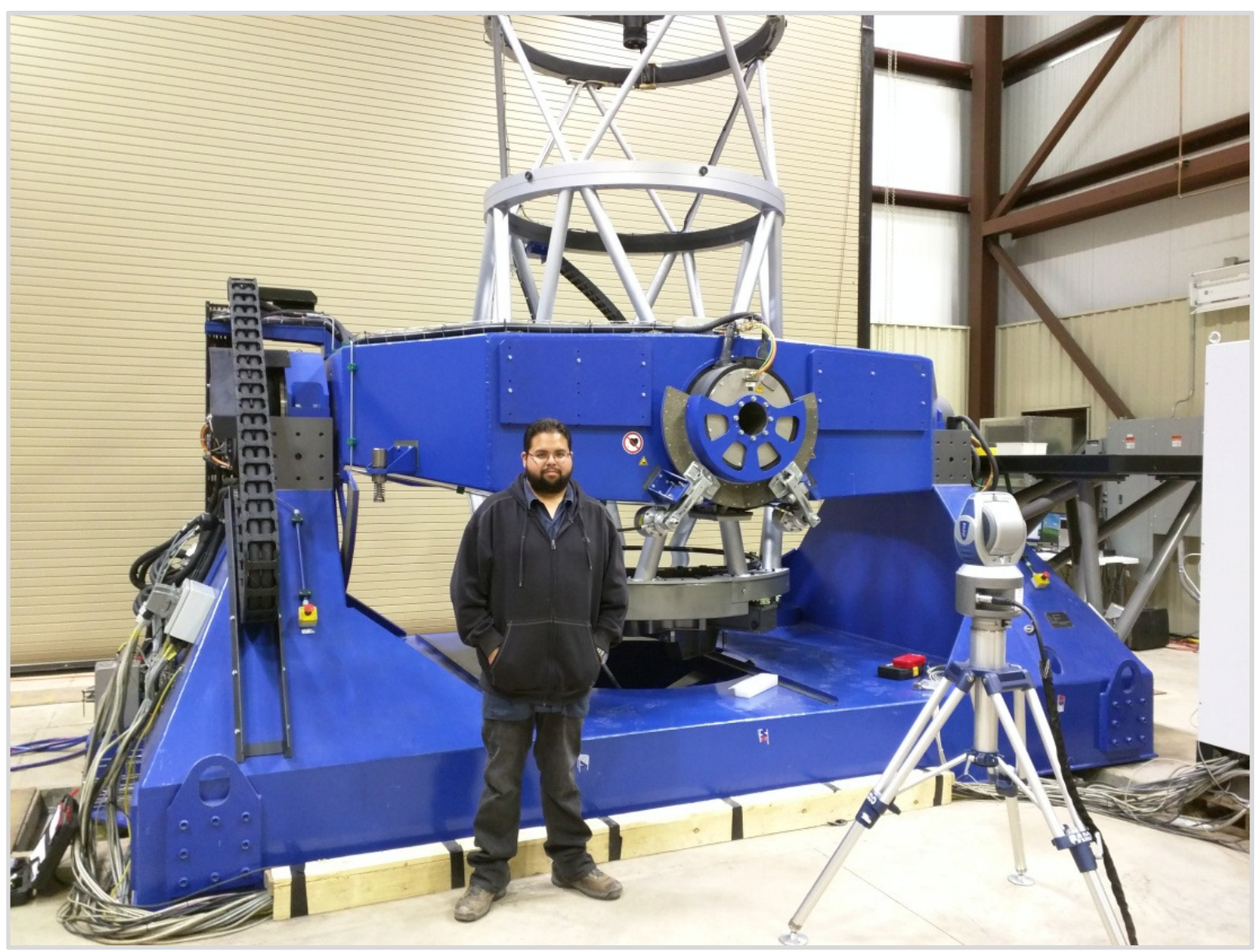

Figure 3: UTM on the kinematic mount in the VCMF. In the foreground is a laser tracking device used to align all the mirrors to the UTM structure in a few hours.

Integration of the Fast Tip-Tilt (see section 2.4 below) system on the adjacent Nasmyth table will be conducted after telescope SAT is completed later this fall. Integration of all software via the Supervisory System (see section 2.8 below) will allow for initial testing of the telescope mount system prior to integration with the Unit Telescope Enclosure in the summer of 2017.

\section{$2.2 \quad$ Unit Telescope Enclosures}

The Unit Telescope Enclosures (UTE) both protect and are used to transport the UTMs on the MROI array and are specified to be repositioned and operating in a few hours ${ }^{5}$. EIE Group is the prime contractor on the design of the UTEs and is working with several subcontractors to complete the fabrication of the first UTE later in 2016 (see figures 4a and b below). After Factory Acceptance Testing (FAT) is completed, we expect to receive the first UTE at the observatory for SAT. We will integrate the UTM and UTE on the array along the West arm during the summer of 2017 for this demonstration. A recent description of the UTE design and fabrication can be seen in Marchiori et al. (2016) ${ }^{6}$. 

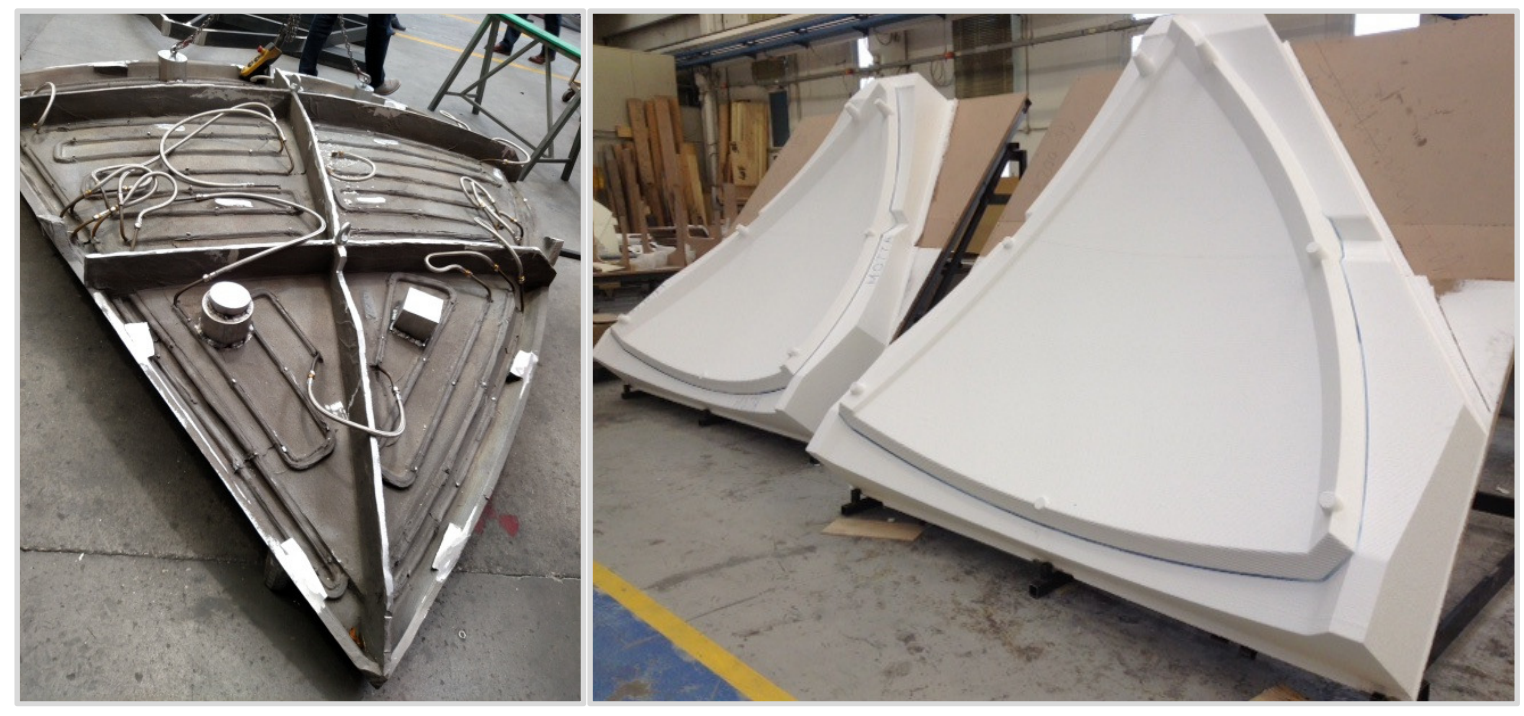

Figure 4a and b: On the left is a mold for manufacturing a portion of the MROI Unit Telescope Enclosure, and on the right are portions of the enclosure.

\subsection{Telescope Optics}

The MROI's zerodur optics perform as an F/2.25 Mersenne system to deliver a collimated $95 \mathrm{~mm}$ diameter beam into the Beam Relay System (BRS). Several years ago 6 sets of primaries, secondaries and tertiaries were purchased, with the secondaries and tertiaries all completed to the required specifications to deliver a system $63 \mathrm{~nm}$ rms wavefront from the telescope when support infrastructure and control of the optics is included. The primaries were under development when our first polishing company went bankrupt during the recession and the work had to stop. We are presently testing the performance of the first UTM using a primary mirror that has reached $52 \mathrm{~nm}$ rms surface, slightly worse than the final required figure. The observatory will shortly be letting a Request for Proposal (RFP) for finishing the primaries for telescopes 2 and 3 to be completed by 2018, in time for first fringes and closure phase/bootstrapping demonstrations. See figure 5 for a picture of the coated primary being installed for Site Acceptance Testing (SAT) of UTM\#1. 


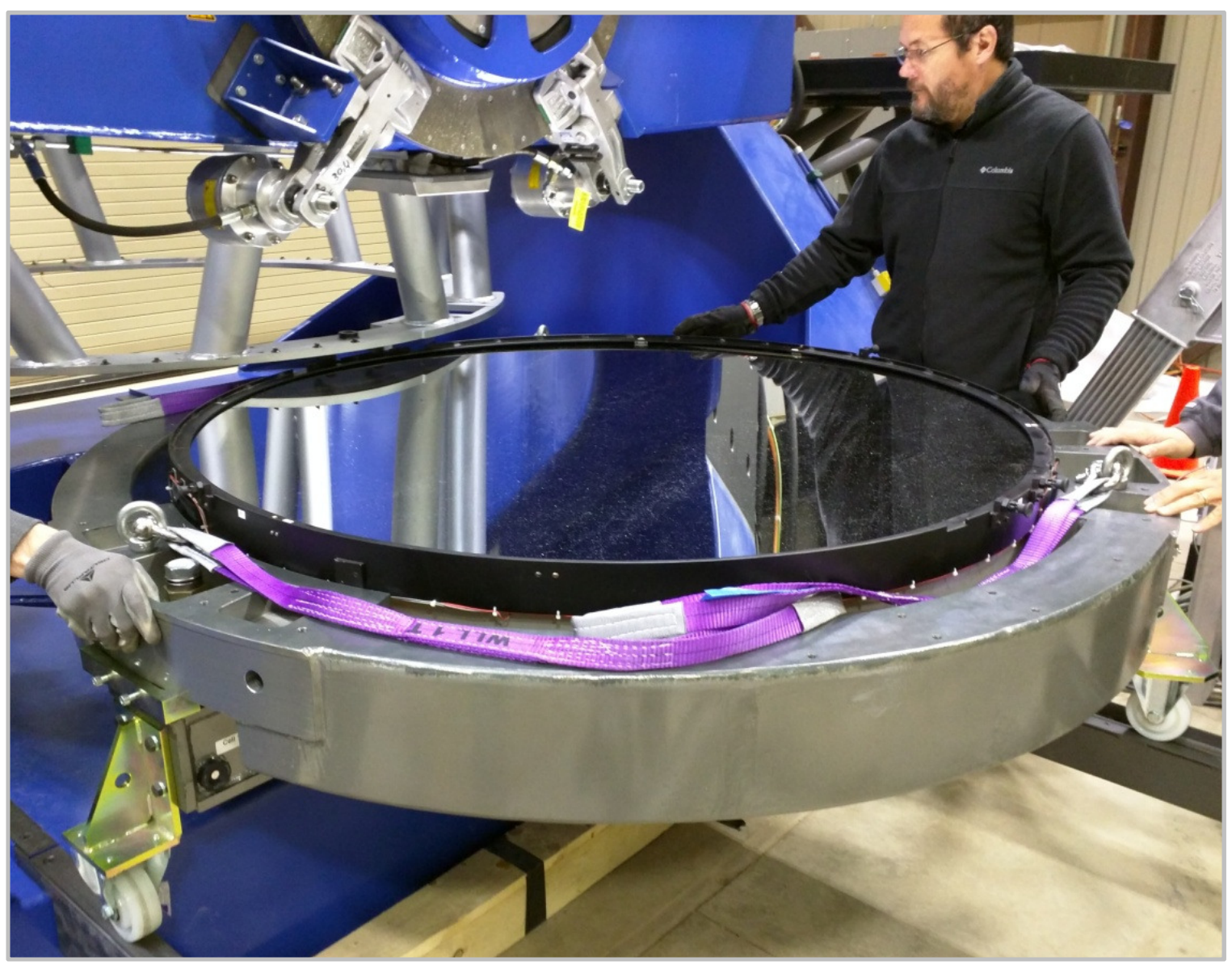

Figure 5: Aluminum coated primary being installed in the first telescope mount in the VCMF.

\subsection{Fast Tip-Tilt and First Light Camera Systems}

The Fast Tip-Tilt (FTT) system works in the optical (either short of 600 or $900 \mathrm{~nm}$ ) to measure the lowest order imperfections of the incoming stellar wavefront at the Nasmyth table and correct them by tipping/tilting the secondary of the telescope. This Cambridge-designed system is reflective and mostly passive, allowing it to easily attain the wavefront and bandwidth requirements of the interferometer system (c.f. Young et al. (2014) ${ }^{7}$ for more information). The FTT system is presently undergoing test at Cambridge and will be deployed at MROI after SAT of the first UTM is completed. Connected to the UTM is the First Light Camera (FLC), which is a temporary narrow-field acquisition/guide camera being used during UTM SAT in conjunction with the Wide-Angle Sensor. After SAT is completed, the FLC will be replaced on the Nasmyth table with the FTT/Narrow-Angle Sensor. See figures $6 a$ and $b$ below for recent photos of the systems under test at Cambridge. 

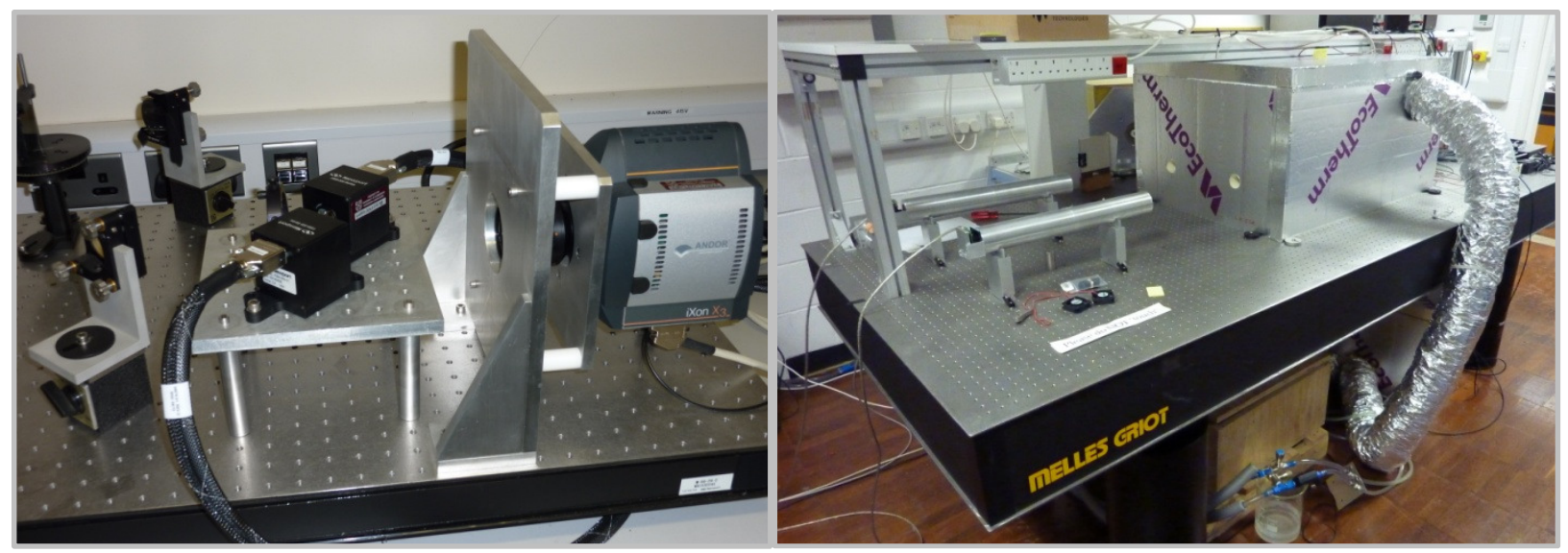

Figure 6a and b: On the left is the Fast Tip-Tilt system, specifically the FTT EMCCD, under test at Cambridge; on the right are the FTT opto-mechanics being tested (in the enclosure) with incoming fake telescope beams in a thermal enclosure to simulate performance on sky.

\subsection{Infrastructure and Beam Relay System}

The array infrastructure and beam relay system (BRS) are important and often overlooked parts of an interferometric instrument. The infrastructure includes the telescope and enclosure pads, which are physically co-located but separated in order to reduce vibrations within the system. Each pad also includes utilities such as power, internet communications and a chiller loop. Presently seven pads are installed on the MROI array, five along the West arm and one each along the East and North arms. These pads do not yet have permanent utilities included, but this infrastructure will be installed incrementally over the next few years. The stellar beams from the telescopes are transported in vacuum (at 0.5 millibars) after passing over the Nasmyth table of the telescope until they exit the delay lines in order to diminish losses associated with open-air beam transport. One set of beam transport vacuum cans with mirrors are installed today at $\mathrm{MROI}$ and are presently undergoing long-term testing for diurnal stability. Initial indications are that pier motion associated with solar heating causes significant beam motion, but the motion is slow and easily tracked with the present system. Full installation of at least three piers will happen by 2020 in support of the efforts between MROI and AFRL. If funding can be secured separately to install more pads and associated infrastructure, and also to purchase a reach stacker for moving telescopes on the array, efforts will be made to install more of the system to increase overall functionality of the array. Below are figures 7 and 8 showing some of the infrastructure and beam relay vacuum cans. 


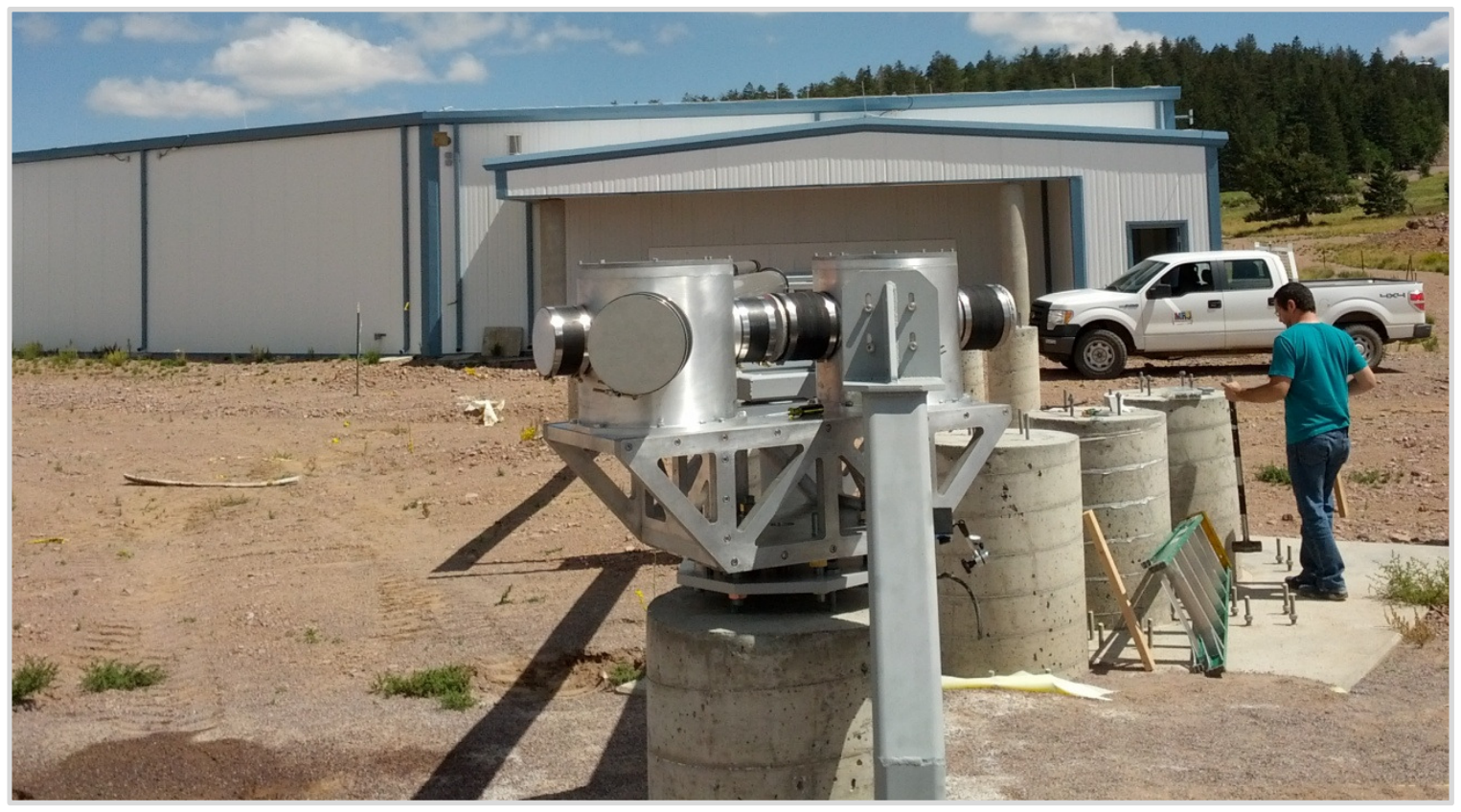

Figure 7: Array infrastructure and optical housings of the Beam Relay System (BRS) looking along the West arm of the interferometer directly into the Beam Combining Facility (BCF).

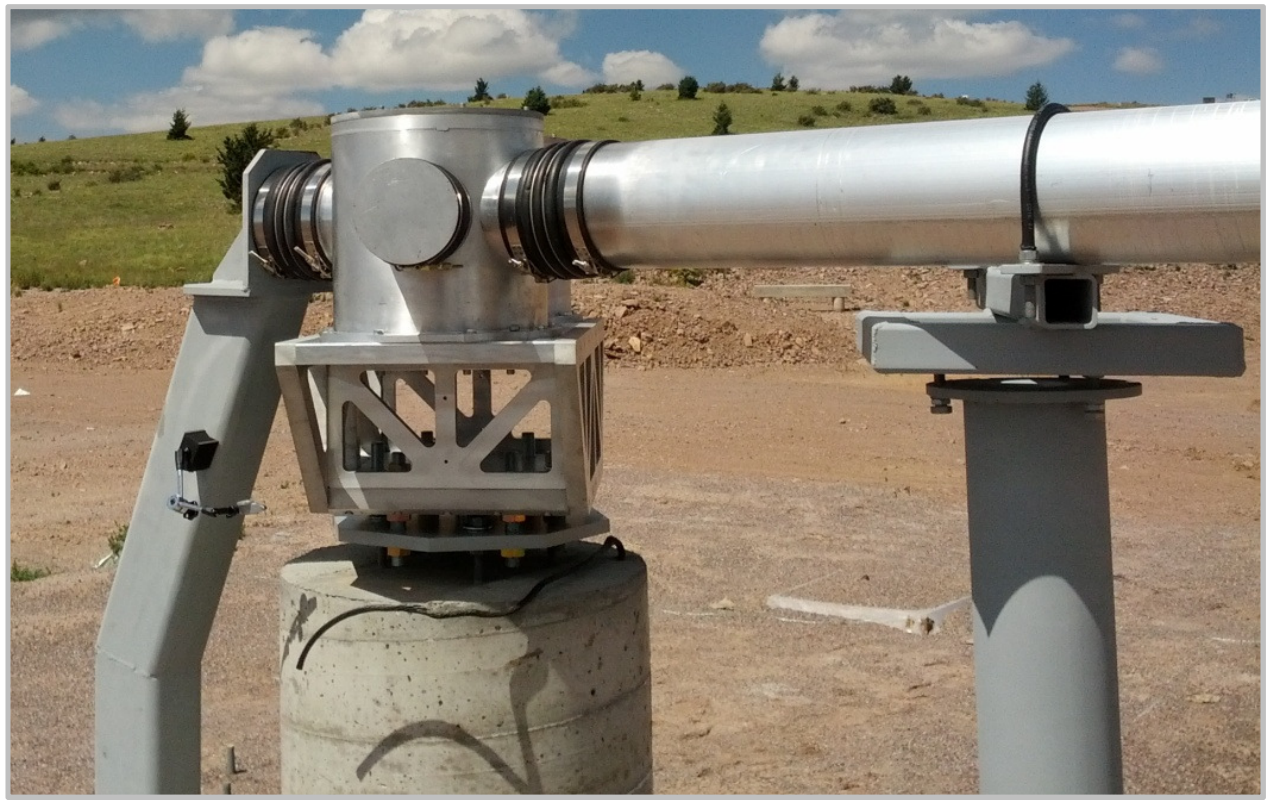

Figure 8: A close-up view of one of the beam relay vacuum cans mounted on its pier. This system has been designed to accept beams from the telescopes and transport them under vacuum into the beam combining facility and to the delay lines. 


\subsection{Delay Lines}

The MROI delay line trolleys and approach to correcting delay are an innovative design developed by Cambridge University ${ }^{8}$. The first delay line trolley was delivered a few years ago and partial site acceptance testing was completed at the observatory in a 100 meter long delay line vacuum pipe. Testing will be completed later this year of both the first and second trolleys for the interferometer. Shortly thereafter, installation of a second delay line vacuum pipe will be undertaken in preparation for first fringes, expected in late 2018. In the interim period, the initial delay line and trolley will be used to characterize the system throughput as well as to perform integration with the supervisory system of the interferometer. Figures 9 and 10 show delay line trolley \#2 under assembly at Cambridge and a view of the first section of delay line vacuum pipe as seen from the inner beam combining room respectively.

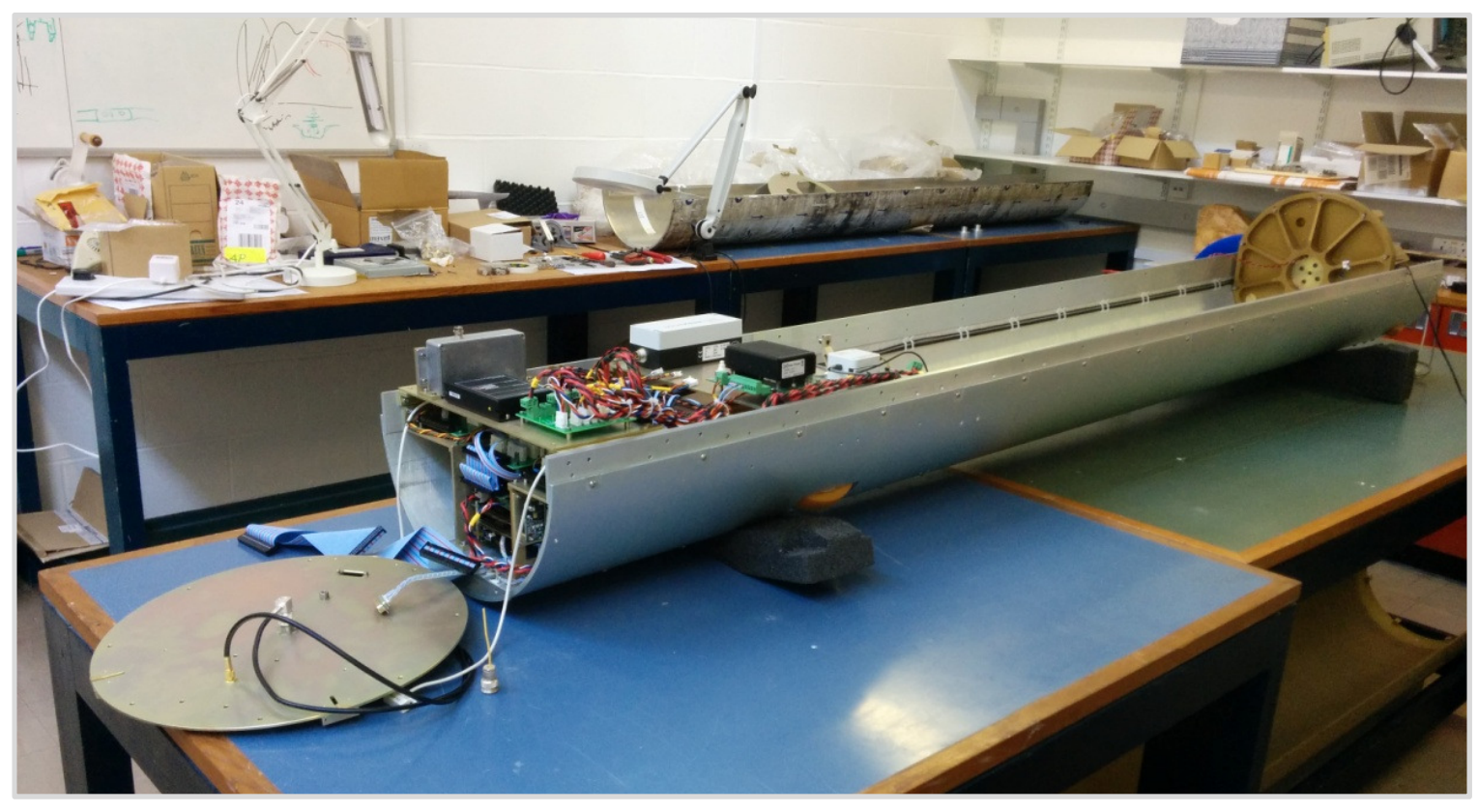

Figure 9: Delay Line trolley \#2 under final assembly at Cambridge in the lab. 


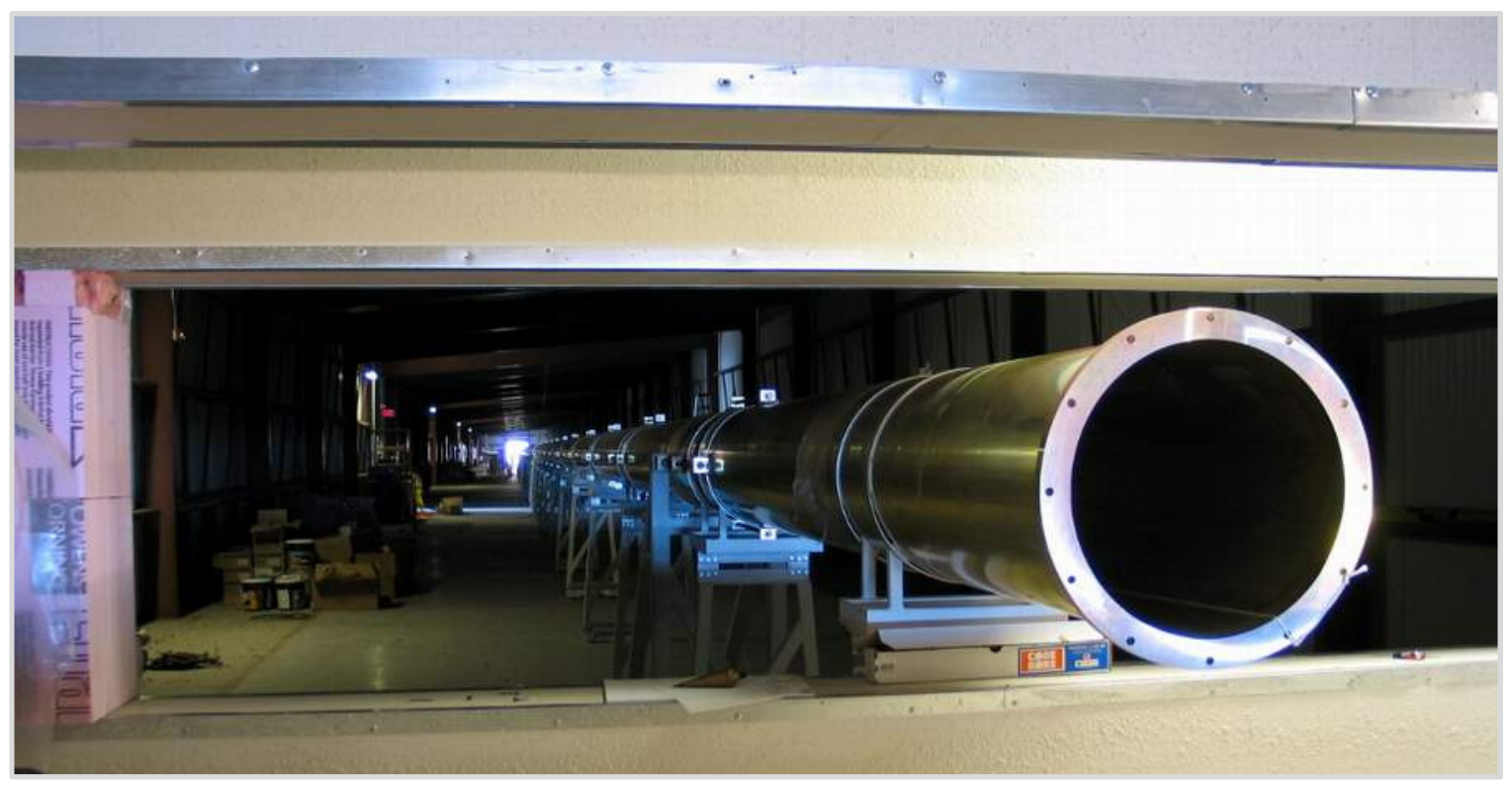

Figure 10: First delay line pipe installed in the delay line portion of the beam combining facility. This pipe was installed to a length of 100 meters and used for initial testing of the first delay line trolley.

\subsection{Fringe Tracker}

The MROI fringe tracker is a nearest-neighbors style near-infrared fringe tracker aptly named ICoNN (Infrared Coherencing Nearest Neighbor) combiner. It had undergone extensive development and testing prior to the loss of MROI funding in 2012. Further information about ICoNN can be found in Jurgenson et al. $(2013)^{9}$ and McCracken et al. (2013) ${ }^{10}$. To date, the ICoNN beam combiner's inner 6 (of the full 10) optical beam trains have all been tested in the lab using small delay lines and an artificial atmosphere to test the ability of the tracking algorithms to follow system perturbations. In preparation for first fringes in late 2018 , ICoNN will be augmented to include off-axis parabolas, filter mechanisms and pinholes in the dewar (figure 11 below) and alignment of the system to the beam combiner using external periscope optics. The last major step in the preparation of ICoNN will be determining whether first-fringes will be undertaken using the current PICNIC detector, or using a new avalanche photodiode SAPHIRA detector from Selex ${ }^{11}$. Initial developments and tests of a SAPHIRA are currently underway at Cambridge and are expected to fundamentally change the approach to interferometric fringe tracking ${ }^{12}$. 


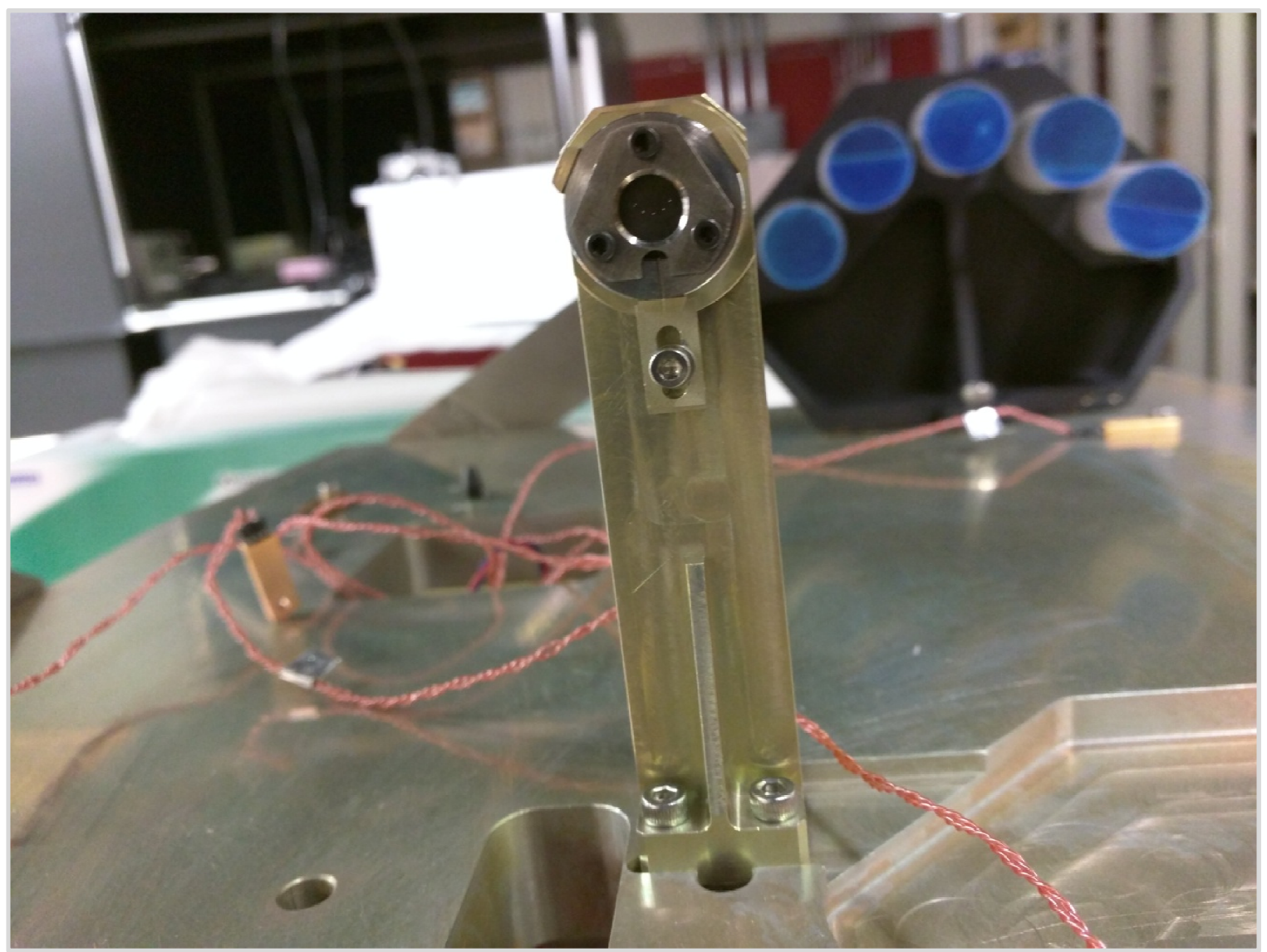

Figure 11: Optical baseplate of ICoNN looking from the first set of off-axis parabolas past the central pinholes toward the second set of off-axis parabolas. Final integration and alignment of all internal components of ICoNN will be completed in the lab during 2017.

\subsection{Supervisory System}

The MROI Supervisory System manages resources using Ethernet over gigabit optical fiber and the TCP/IP protocol to form a unified and coherent distributed system. Applications are developed and tested independently and integrated into that framework by specifying spreadsheets describing the public interface of that application. These spreadsheets are then input to a code generation framework that generates a layer of client/server software and database entries that describe the application, its public interfaces, and the monitor data it produces. All subsystems of the interferometer are under development and have reached various stages of completion, with the expectation of most systems being tested in situ and all coming online within the 5-year period. More information about the MROI software architecture can be found in Farris et al. (2010) $)^{13}$. Below we show a diagrammatic representation of the portion of the Supervisory System related to the Unit Telescope. 


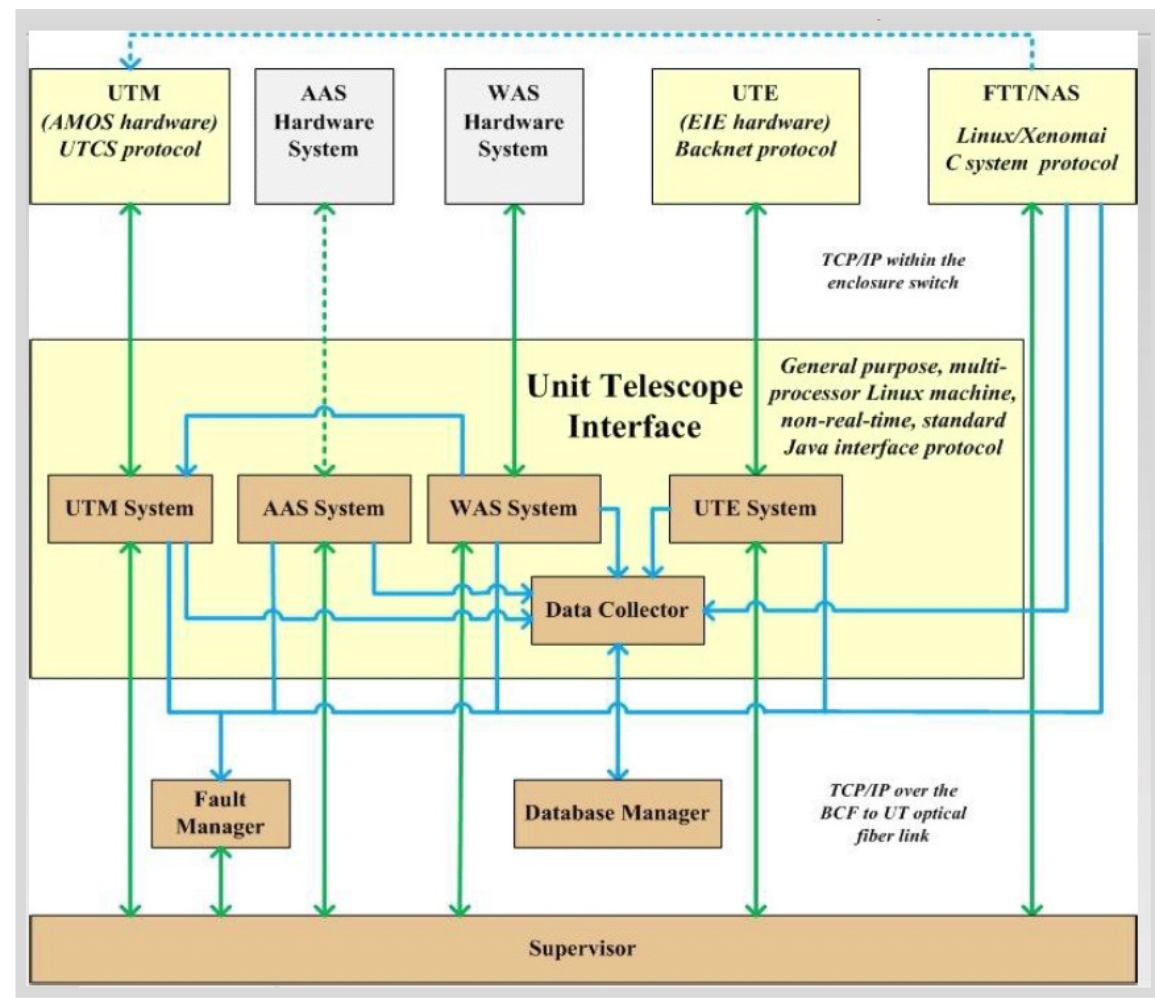

Figure 12: A schematic of the Unit Telescope portion of the Supervisory System. Each of the more than 20 subsystems of the interferometer has a similarly designed and detailed control infrastructure needed to operate the overall system.

\subsection{Future Development and Conclusions}

Between today and 2020 the MROI will attain both first fringes and closure phase to demonstrate its capability for supporting the AFRL SSA mission, as well as superior capabilities on astrophysical targets. We anticipate a few more professional and several more student positions to be available in the near future to assist in completing this first phase of MROI. After this initial phase is complete and capabilities are fully demonstrated, the project will seek peer-reviewed funding to expand to the full 10telescope facility. The system architects and project scientist will be developing a white paper to enumerate the detailed capabilities of the facility in the near and longer term. Investigators interested in learning more about the facility's capabilities or in collaborating or developing instruments should contact the project office at NMT for further information. 


\section{Acknowledgements:}

The MROI is currently funded under AFRL Cooperative Agreement FA9453-15-2-0086 titled "Amplitude Interferometer Research for Geosynchronous Earth Orbit (GEO) Space Situational Awareness (SSA)". We further wish to thank the Congressional Delegation of the State of New Mexico for their ongoing, generous support of the project. Our Cambridge collaborators additionally wish to acknowledge STFC in the UK for support specifically for their work on the Saphira detector.

\section{References:}

[1] Young, J. S. et al. "The MROI's capabilities for imaging geosynchronous satellites", in "Optical and Infrared Interferometry III", Proc. of SPIE, vol. 8445, (2012).

[2] Santoro, F. et al., "Final mechanical and opto-mechanical design of the Magdalena Ridge Observatory Interferometer", in "Optical and Infrared Interferometry III", Proc. of SPIE, vol. 8445, (2012).

[3] Buscher, D. F., Creech-Eakman, M. Farris, A., Haniff, C. A., and Young, J. S., "The Conceptual Design of the Magdalena Ridge Observatory Interferometer", Journal of Astronomical Instrumentation, vol. 2, issue 2, (2013).

[4] Pirnay, O. et al., "Design of the unit telescopes of the MROI", in "Optical and Infrared Interferometry", Proc. of SPIE, vol. 7013, (2008).

[5] Busatta, A., et al., "Innovative enclosure dome/observing aperture system design for the MROI", in "Modern Technologies in Space- and Ground-based Telescopes and Instrumentation", Proc. of SPIE, vol. 7739, (2010).

[6] Marchiori, G. et al., "The MROI Array telescopes: the relocatable enclosure domes", in "Groundbased and Airborne Telescopes VI", Proc. of SPIE, vol. 9906, (2016).

[7] Young, J. S., et al., "The performance of the MROI fast tip-tilt correction system", in "Optical and Infrared Interferometry IV", Proc. of SPIE, vol. 9146, (2014).

[8] Fisher, M. et al., "Design of the MROI delay line optical path compensator", in "Optical and Infrared Interferometry II", Proc. of SPIE, vol. 7734, (2010).

[9] Jurgenson, C. A. et al., "ICoNN - The Infrared Coherencing Nearest Neighbor Tracker", Journal of Astronomical Instrumentation, vol. 2, issue 1, (2013).

[10] McCracken, T. et al., "Open-loop phase shifting for fast acquisition of interferograms at low light levels", Applied Optics, vol. 52, issue 20, p 4922, (2013).

[11] Finger, G., et al., "Evaluation and optimization of NIR HgCdTe avalanche photodiode arrays for adaptive optics and interferometry", in "High Energy, Optical and Infrared Detectors for Astronomy V", Proc. of SPIE, vol. 8453, (2012). 
[12] Buscher, D. F. et al., "Progress towards photon-counting infrared arrays for interferometry", in "Optical and Infrared Interferometry and Imaging V", Proc. of SPIE, vol. 9907, (2016).

[13] Farris, A. et al., "Software architecture of the Magdalena Ridge Observatory Interferometer", in "Software and Cyberinfrastructure for Astronomy", Proc. of SPIE, vol. 7740, (2010). 